\section{Use of resuscitative endovascular balloon occlusion of the aorta in a patient with gastrointestinal bleeding}

\author{
Jungyoup Lee, Kyuseok Kim, You Hwan Jo, Jae Hyuk Lee, Joonghee Kim, \\ Heajin Chung, Ji Eun Hwang
}

Department of Emergency Medicine, Seoul National University Bundang Hospital, Seongnam, Korea

Resuscitative endovascular balloon occlusion of the aorta (REBOA) was developed for controlling intra-abdominal arterial bleeding before definitive bleeding control, and is commonly used in patients with ruptured abdominal aortic aneurysms. Although there is limited evidence for other uses of REBOA, we used REBOA in a patient with massive gastrointestinal bleeding. A 53-yearold man with hematochezia was admitted to our emergency department with an initial systolic blood pressure (SBP) of $83 \mathrm{mmHg}$. His SBP decreased to $40 \mathrm{mmHg}$ in 10 minutes despite rapid fluid infusion. We decided to resuscitate the patient with REBOA in the emergency department and then move him to an intervention room after stabilization. After aortic occlusion, SBP abruptly increased from 57 to $108 \mathrm{mmHg}$, and the patient could be transferred to an intervention room. The patient was admitted to intensive care, but died of massive rebleeding 24 hours after admission to the emergency department.

Keywords Resuscitation; Shock; Aorta; Hemorrhage; Intestines

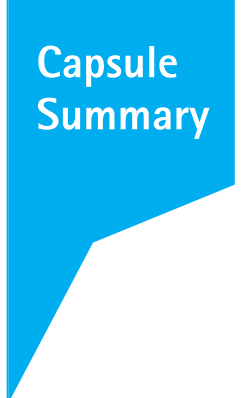

What is already known

Resuscitative endovascular balloon occlusion of the aorta is used in patient with ruptured abdominal aortic aneurysms before definitive bleeding control.

What is new in the current study

We used resuscitative endovascular balloon occlusion of the aorta in patient with massive gastrointestinal bleeding with rapid improvement in systolic blood pressure. However, the patient subsequently died from rebleeding 24 hours later.
eISSN: $2383-4625$

Received: 2 October 2015

Revised: 15 November 2015

Accepted: 16 November 2015

Correspondence to: Kyuseok Kim Department of Emergency Medicine, Seoul National University Bundang Hospital, 82 Gumi-ro 173beon-gil, Bundang-gu, Seongnam 13620, Korea E-mail:dremkks@snubh.org

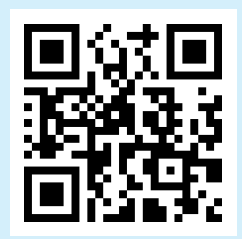

How to cite this article:

Lee J, Kim K, Jo YH, Lee JH, Kim J, Chung H, Hwang JE. Use of resuscitative endovascular balloon occlusion of the aorta in a patient with gastrointestinal bleeding. Clin Exp Emerg Med 2016;3(1):55-58.

This is an Open Access article distributed under the terms of the Creative Commons Attribution Non-Commercial License (http:// creativecommons.org/licenses/by-nc/3.0/). 


\section{INTRODUCTION}

Resuscitative endovascular balloon occlusion of the aorta (RE$\mathrm{BOA}$ ) can be used to control intra-abdominal arterial bleeding. A REBOA device is inserted into the common femoral artery and placed in the aorta to block the distal flow of the aorta. It is used for temporary control of active bleeding in patients with ruptured abdominal aortic aneurysms before definitive hemorrhage control. ${ }^{1}$ This intra-aortic device was first described in patients with uncontrolled intra-abdominal bleeding in the Korean War. ${ }^{2}$ In the field of thoracoabdominal trauma, a case series and clinical study have been reported. ${ }^{3,4}$ Theoretically, REBOA can control all kinds of arterial bleeding in the intra-abdominal organs. However, there is limited evidence for the use of REBOA in cases besides those involving ruptured abdominal aortic aneurysms. We present a patient with uncontrolled and obscure gastrointestinal (GI) bleeding who underwent REBOA in our emergency department (ED).

\section{CASE REPORT}

A 53-year-old man with alcohol-induced liver cirrhosis (ChildPugh class C) was admitted to another hospital due to hematochezia. The patient underwent esophagogastroduodenoscopy and colonoscopy at the other hospital; however, the active bleeding site was not detected. When the patient was admitted to our ED, hematochezia was still observed, and his mental status changed from alertness to stupor. Initial vital signs were as follows: systolic blood pressure (SBP), $83 \mathrm{mmHg}$; diastolic blood pressure, 46 $\mathrm{mmHg}$; heart rate, 135 beats/min; and respiratory rate, 20 cycles/ min. Although a multilumen access catheter (Arrow; Teleflex Medical, Research Triangle Park, NC, USA) was inserted in the internal jugular vein and packed red blood cells were infused by a rapid infusion pump (Level1; Smiths Medical, Dublin, OH, USA) with crystalloid and norepinephrine, the SBP decreased to 40 $\mathrm{mmHg}$ and the mental condition also deteriorated gradually. Initial laboratory results were as follows: hemoglobin, $5.1 \mathrm{~g} / \mathrm{dL}$; platelets, 30,000/ $\mu \mathrm{L}$; total bilirubin, $2 \mathrm{mg} / \mathrm{dL}$; albumin, $1.3 \mathrm{~g} / \mathrm{dL}$; and prothrombin time/international normalized ratio, 4.74 . We thought that the patient was about to experience a cardiac arrest. Therefore, we decided to try and resuscitate the patient with REBOA first. After verbal consent from the patient's family to use the device, the right common femoral artery was punctured under sonographic guidance and a 12-French introducer was placed using the Seldinger technique. A metal guidewire was inserted into the aorta via the introducer and the REBOA was inserted over the metal guidewire. After we checked the portable X-ray results and confirmed that the REBOA had been placed in the de-



Fig. 1. Radiograph of resuscitative endovascular balloon occlusion of the aorta.

scending thoracic aorta, we inflated the balloon with normal saline (Fig. 1). After occlusion of the aorta, the SBP abruptly increased from 57 to $108 \mathrm{mmHg}$, and the patient could be transferred to the vascular intervention room. The REBOA was inflated for 15 minutes and deflated gradually during angiography. After removal of the REBOA, his vital signs remained stable for several hours. Angiography did not detect the active arterial bleeding site and the patient was admitted to the intensive care unit. Esophagogastroduodenoscopy was performed in the intensive care unit, and a Dieulafoy ulcer and duodenal varix without active bleeding were detected in the second part of the duodenum. The Dieulafoy ulcer was clipped by hemoclips and duodenal varix embolization was performed. Although vital signs remained stable under vasopressor treatment, hemoglobin levels decreased continuously, and transfusion was needed during the admission period. The bleeding source was still obscure; hypovolemic shock and cardiac arrest occurred 24 hours after admission to the ED. A total of 19 packed red blood cell units were transfused until cardiac arrest. We tried to resuscitate the patient with cardiopulmonary resuscitation, but failed. 


\section{DISCUSSION}

We describe a patient with massive Gl bleeding who underwent REBOA in our ED. Previously, patients with massive GI bleeding died before admission to the ED and a definitive bleeding control intervention. Therefore, we speculated that if patients with massive $\mathrm{Gl}$ bleeding, who are unresponsive to transfusion, were stabilized by REBOA, there would be a better chance of definitively controlling the bleeding. Our patient was not stabilized by rapid transfusion in the ED, and we could not resuscitate the patient when he experienced cardiac arrest. Although there is no evidence of the use of REBOA in Gl bleeding, we used this as a bridging therapy because other resuscitation options were unavailable. As a result, the patient was stabilized by REBOA in the ED; however, the active bleeding site was not detected on angiography. During angiography, the REBOA was removed carefully and stable vital signs were maintained. Hence, we presumed that the active bleeding source was controlled temporarily with the aortic occlusion. Thus, although there is no evidence of the use of REBOA to control massive Gl bleeding, further research could justify the use of REBOA as a bridging therapy.

REBOA has a two-fold mechanism in the aorta. First, REBOA controls arterial bleeding in cases of noncompressible intra-abdominal hemorrhage. Second, REBOA augments cerebral and myocardial perfusion because the distal blood flow is blocked by the balloon. REBOA can be used instead of open aortic clamping. Moreover, REBOA is less invasive and easy to perform in emergencies.

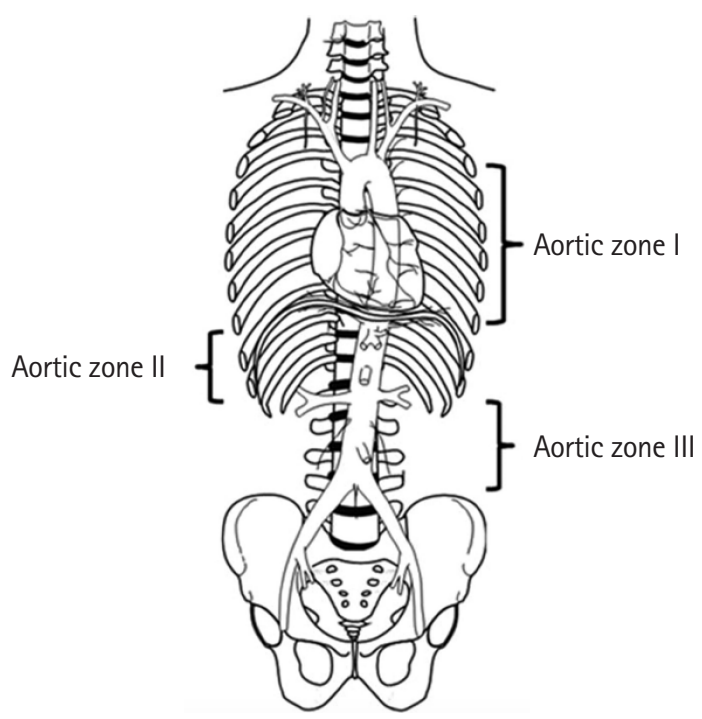

Fig. 2. Division of aortic zones. Zone I extends from the origin of the subclavian artery to the celiac artery, zone II extends from the celiac artery to the origin of the renal artery, and zone III extends from the lowest renal artery to the aortic bifurcation. ${ }^{3}$
Actually, we adjusted REBOA within 15 minutes without acute complications.

The placement of REBOA in the aorta should be decided prior to insertion. We defined the levels of the aorta according to Stannard et al. ${ }^{5}$ (Fig. 2). ${ }^{3}$ In this case, we decided to place the REBOA in zone I because the site of the bleeding was unknown. A balloon in zone I blocks arterial blood flow to the celiac trunk, superior mesenteric artery, and inferior mesenteric artery. Therefore, theoretically, all the arterial GI bleeding can be controlled. For isolated pelvic bone fractures with active bleeding, REBOA is placed in zone III, blocking the common iliac artery.

REBOA can have several complications. First, in zone I REBOA, prolonged ballooning could result in bowel ischemia because the entire arterial blood flow to the $\mathrm{Gl}$ tract is blocked. However, there are no clinical data on aortic ballooning time and bowel ischemia. There was one large animal study on REBOA and bowel ischemia. In the study, bowel ischemia occurred in one out of 8 pigs after 60 minutes of aortic ballooning. ${ }^{6}$ Second, REBOA is inserted into the common femoral artery over the 12-French introducer. If the introducer is inserted into the superficial femoral artery, which is smaller than the common femoral artery, limb ischemia and arteriovenous fistula could occur. Puncture site hematoma and infection are also possible complications. Although we think that REBOA can be used in patients with active GI bleeding temporarily, there is no evidence that REBOA can be useful in this situation. However, if patients with $\mathrm{Gl}$ bleeding cannot be stabilized in the ED using other methods, REBOA could be a useful bridging therapy in spite of the possible complications.

\section{CONFLICT OF INTEREST}

No potential conflict of interest relevant to this article was reported.

\section{REFERENCES}

1. Mayer $D$, Pfammatter T, Rancic $Z$, et al. 10 years of emergency endovascular aneurysm repair for ruptured abdominal aortoiliac aneurysms: lessons learned. Ann Surg 2009;249:510-5.

2. Hughes $\mathrm{CW}$. Use of an intra-aortic balloon catheter tamponade for controlling intra-abdominal hemorrhage in man. Surgery 1954;36:65-8.

3. Brenner ML, Moore $\sqcup$, DuBose JJ, et al. A clinical series of resuscitative endovascular balloon occlusion of the aorta for hemorrhage control and resuscitation. J Trauma Acute Care Surg 2013;75:506-11.

4. Norii T, Crandall C, Terasaka Y. Survival of severe blunt trauma 
patients treated with resuscitative endovascular balloon occlusion of the aorta compared with propensity score-adjusted untreated patients. J Trauma Acute Care Surg 2015;78:721-8.

5. Stannard A, Eliason JL, Rasmussen TE. Resuscitative endovascular balloon occlusion of the aorta (REBOA) as an adjunct for hemorrhagic shock. J Trauma 2011;71:1869-72.

6. Morrison JJ, Ross JD, Houston R 4th, Watson JD, Sokol KK, Rasmussen TE. Use of resuscitative endovascular balloon occlusion of the aorta in a highly lethal model of noncompressible torso hemorrhage. Shock 2014;41:130-7. 